OPEN

SUBJECT AREAS:

NANOPHOTONICS AND

PLASMONICS

QUANTUM OPTICS

Received

13 December 2013

Accepted

8 April 2014

Published

24 April 2014

Correspondence and requests for materials should be addressed to T.A. (takao@waseda.

\title{
Efficient Single-Mode Photon-Coupling Device Utilizing a Nanofiber Tip
}

\author{
Sho Chonan, Shinya Kato \& Takao Aoki
}

Department of Applied Physics, Waseda University, Okubo 3-4-1, Shinjuku, Tokyo, Japan.

Single-photon sources are important elements in quantum optics and quantum information science. It is crucial that such sources be able to couple photons emitted from a single quantum emitter to a single propagating mode, preferably to the guided mode of a single-mode optical fiber, with high efficiency. Various photonic devices have been successfully demonstrated to efficiently couple photons from an emitter to a single mode of a cavity or a waveguide. However, efficient coupling of these devices to optical fibers is sometimes challenging. Here we show that up to $38 \%$ of photons from an emitter can be directly coupled to a single-mode optical fiber by utilizing the flat tip of a silica nanofiber. With the aid of a metallic mirror, the efficiency can be increased to $76 \%$. The use of a silicon waveguide further increases the efficiency to $87 \%$. This simple device can be applied to various quantum emitters.

ingle-photon sources are indispensable in many applications in optical quantum information science, including quantum key distribution ${ }^{1}$ and linear-optical quantum computing ${ }^{2,3}$. In such applications, it is required to couple photons emitted from a single quantum emitter to a single propagating mode, preferably to the guided mode of a single-mode optical fiber, with high efficiency. A variety of quantum emitters, such as atoms $s^{4,5}$, ions ${ }^{6}$, dye molecules ${ }^{7}$, semiconductor quantum dots ${ }^{8,9}$, and defect centers in diamond crystals $^{10}$, have been used in single-photon sources.

A cavity quantum electrodynamics (QED) system enables one to efficiently couple photons from such an emitter to a single spatial mode ${ }^{11}$. When an emitter is placed in a cavity, the selective coupling of emitted photons into the cavity mode can be achieved owing to the enhancement of spontaneous emission (the Purcell effect) ${ }^{12}$. However, further coupling of the photons collected by the cavity to a single-mode optical fiber results in losses, which greatly reduce the total coupling efficiency. The use of a fiber-coupled optical microcavity ${ }^{13}$ is a more elaborate way to efficiently couple photons from an emitter to a single-mode optical fiber. The efficient coupling of a fiber input/output mode to an atom in a cavity has been demonstrated using toroidal ${ }^{14}$ and bottle ${ }^{15}$ micro- $^{-}$ resonators coupled to a tapered optical fiber with low losses. A fiber-coupled cavity QED system with a quantum dot embedded in a semiconductor microdisk has also been realized ${ }^{16}$. Recent development of fiber Fabry-Perot cavities with small mode volumes and high finesses ${ }^{17}$ has sparked realizations of fiber Fabry-Perot cavity QED systems with ions ${ }^{18,19}$, quantum $\operatorname{dots}^{20}$, and NV centers in diamond ${ }^{21,22}$.

On the other hand, efficient coupling of photons from an emitter to a single spatial mode can also be achieved by using an optical waveguide. Near-unity coupling efficiency of photons from an emitter embedded in a photonic crystal waveguide has been theoretically predicted ${ }^{23}$ and experimentally demonstrated with quantum $\operatorname{dots}^{24,25}$. It has also been proposed to use guided surface plasmons on metallic nanowires to achieve near-unity coupling efficiency ${ }^{26,27}$, and efficient coupling of photons from quantum dots to surface plasmons on silver nanowires has been demonstrated ${ }^{28}$. However, further coupling of photons collected in these systems to single-mode optical fibers again results in additional losses.

These additional losses in coupling to single-mode fibers can be minimized by the use of a subwavelengthradius cylindrical silica waveguide (i.e., a silica nanofiber) ${ }^{29}$. It has been theoretically shown that efficient coupling of photons to the guided mode of a nanofiber can be achieved when an emitter is located near the surface of the side of the nanofiber ${ }^{30,31}$. The fundamental guided mode of a silica nanofiber can be transferred to the guided mode of a standard single-mode fiber through an adiabatically tapered region with low losses ${ }^{32-34}$. Therefore, the efficient coupling of photons from an emitter to a single-mode optical fiber can be realized with such systems. Recent experimental progress has demonstrated the efficient interaction between the evanescent field of the nanofiber-guided mode and atoms ${ }^{35-40}$. Direct collection of photons emitted from quantum dots and nitrogenvacancy centers in diamond placed on the nanofiber surface to the nanofiber-guided mode has also been reported $^{41-43}$. Efficient coupling of photons from emitters embedded in semiconductor membranes or waveguides 
a

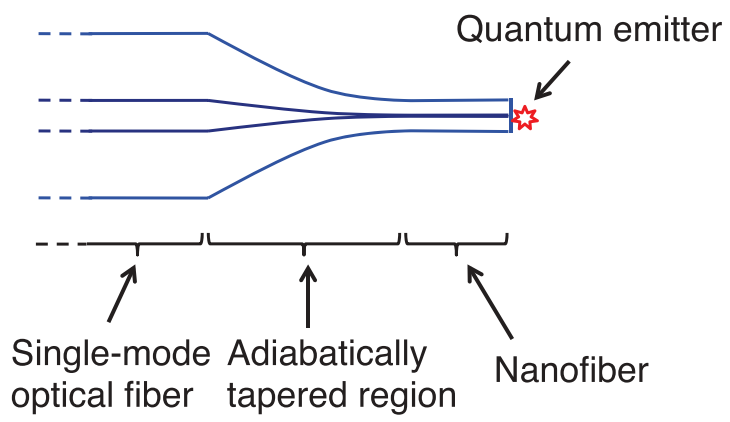

b

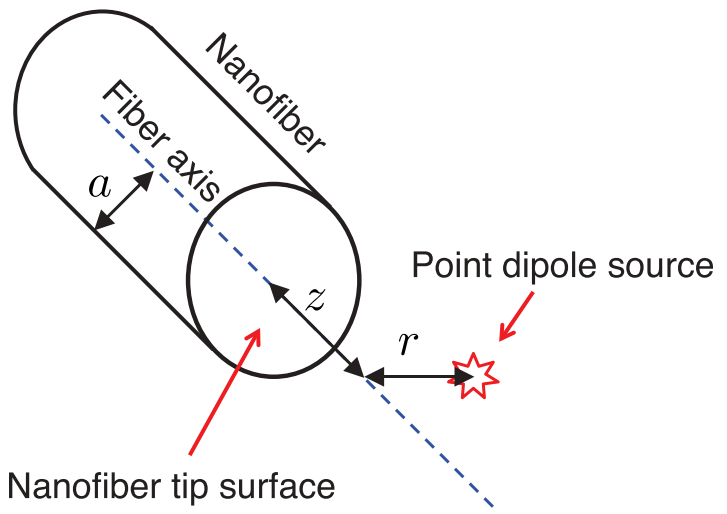

C
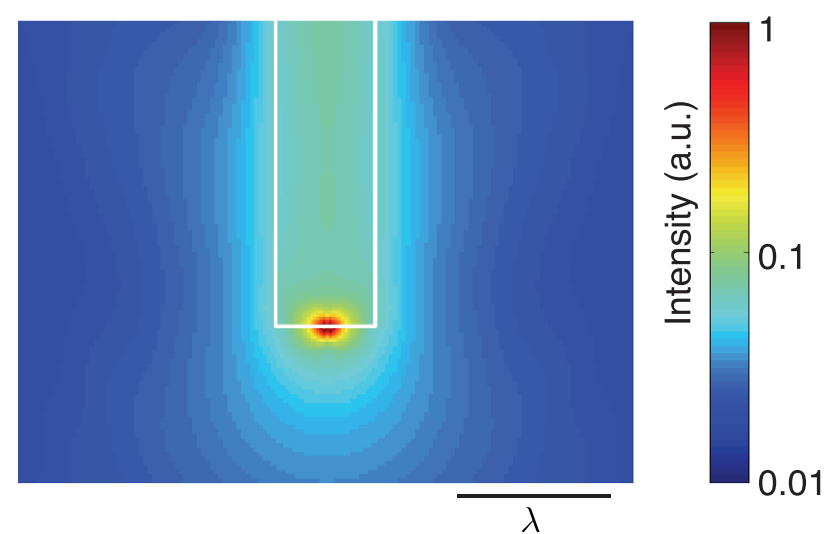

Figure 1 Nanofiber-tip-based coupling of photons from a single quantum emitter to a single-mode optical fiber. (a), Schematic of a nanofiber with a flat tip connected to a standard single-mode optical fiber via an adiabatically tapered region. (b), Simulation geometry. $a$ is the radius of the nanofiber, and $z$ and $r$ are the axial and radial distances from the center of the nanofiber tip surface to the point dipole source, respectively. (c), Calculated intensity of the radiation from a point dipole source located at the center of the nanofiber tip surface $(r=z=0)$ for the case of a nanofiber radius of $a=0.32 \lambda$. The white line indicates the surface of the nanofiber.

has also been theoretically predicted ${ }^{44,45}$ and experimentally demonstrated $^{46}$. These devices utilize the evanescent field of the nanofiberguided mode by placing the emitter near the surface of the side of the nanofiber. Here we propose a device utilizing a nanofiber tip. Because of the interference between the radiation from the emitter and that from the emitter's mirror image on the tip surface, the coupling efficiency is even higher than in the case in which the emitter is embedded in a continuous nanofiber, or the case in which the emitter is placed near the side of the nanofiber. We numerically simulate the coupling efficiency of radiation from a point dipole source located in the vicinity of the tip of a nanofiber to the fundamental guided mode of a nanofiber and obtain an efficiency as high as 38\%. When flat and spherical mirrors are placed in front of the nanofiber tip, the efficiencies reach values as high as $73 \%$ and $76 \%$, respectively. The use of a silicon waveguide further increases the efficiency to $87 \%$.

\section{Results}

Figure 1a shows a schematic of the proposed device. A silica nanofiber with a flat tip is connected to a standard single-mode optical fiber through an adiabatically tapered region. Such a structure can be fabricated by cutting a tapered optical fiber ${ }^{29}$ at its waist. We calculate the coupling efficiency of the radiation from a point dipole source to the fundamental guided mode of the nanofiber, with a geometry shown in Fig. 1b. Figure 1c shows the calculated intensity of radiation for the case of $r=z=0$ and $a=0.32 \lambda$, which clearly shows that the radiation from the point dipole source is efficiently guided to the nanofiber. Note that the fundamental guided mode of the nanofiber
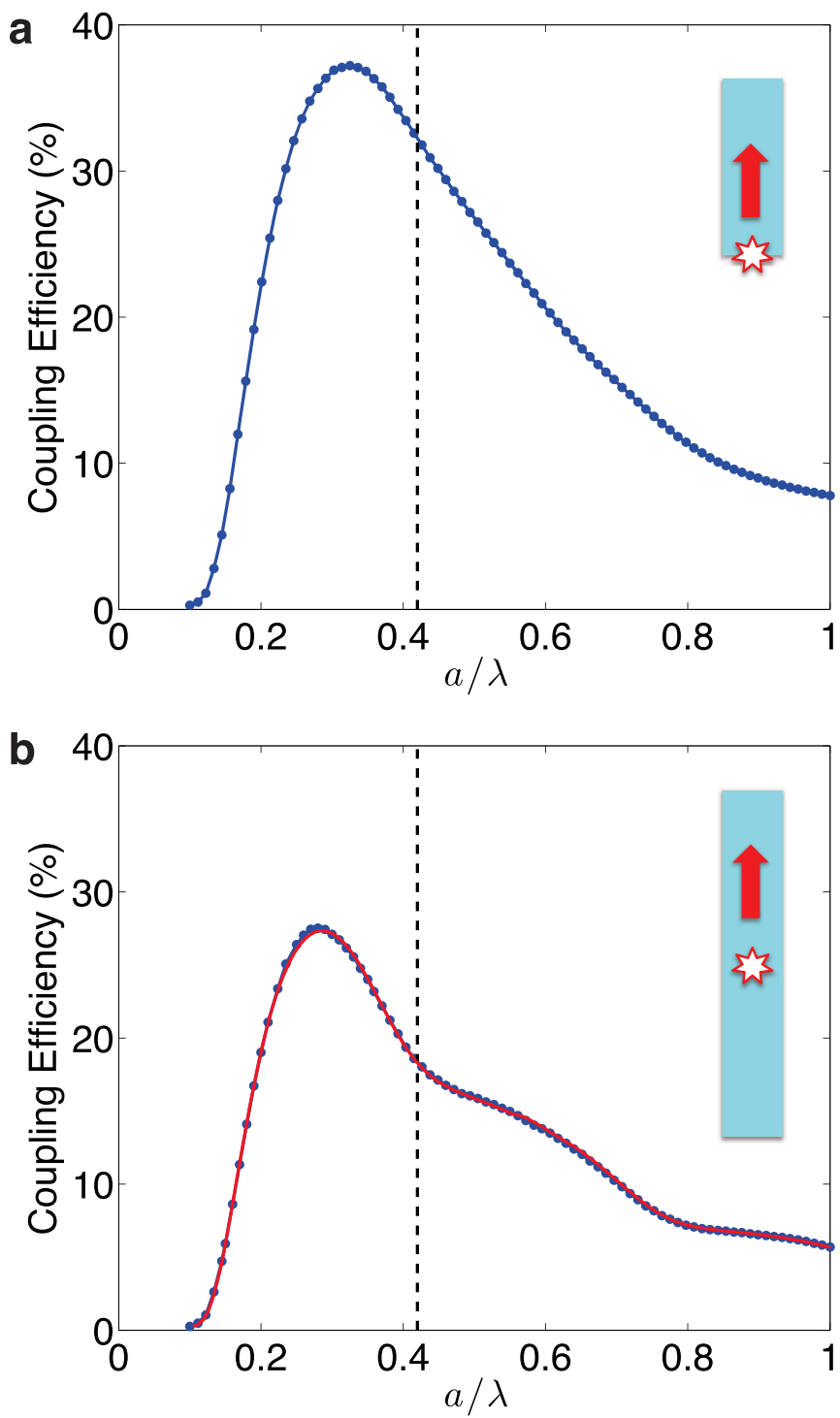

Figure $2 \mid$ Coupling efficiency of radiation from a point dipole source located on the fiber axis $(r=0)$ to the fundamental guided mode of the nanofiber. a, Dependence of the coupling efficiency on the nanofiber radius $a$ for the case of a nanofiber tip with a tip-source distance $z=0$. The inset shows a schematic of the geometry. The vertical dashed line indicates the single-mode cut-off radius. b, Same as a, for the case of a continuous nanofiber. The red solid line shows the coupling efficiency calculated based on the model in Ref. 31. 
is transferred to the guided mode of the single-mode fiber without coupling to the higher-order or radiation modes through the adiabatically tapered region ${ }^{32-34}$. The adiabatic limit for the modal conversion in the tapered region is given by $\Omega \ll r\left(\beta_{1}-\beta_{2}\right) /(2 \pi)$, where $\Omega, r, \beta_{1}, \beta_{2}$ respectively denote the local taper angle, the local fiber radius, and the propagation constants of the fundamental and the first excited modes. In this limit, the mode conversion in the tapered region is adiabatic, and there are no modal coupling losses. Therefore, the coupling efficiency calculated here can be interpreted as the coupling efficiency from the source to the guided mode of a single-mode fiber.

First, we calculate the coupling efficiency for various fiber radii, with the location of the point dipole source fixed at the center of the nanofiber tip surface $(z=r=0)$. Figure 2a shows the dependence of the coupling efficiency on the nanofiber radius $a$ for the case of radial source polarization. A maximum coupling efficiency of $37 \%$ is obtained at $a=0.32 \lambda$, where $\lambda$ is the vacuum wavelength of light. Note that the single-mode cut-off radius is $a=$ $0.42 \lambda$. The obtained efficiency is considerably higher than that for the case in which the source is placed on the surface of a continuous nanofiber ${ }^{30}$. In contrast, we obtain zero coupling efficiency for any value of $a$ for the case of axial source polarization, which reflects the fact that the electric field of the fundamental $\left(\mathrm{HE}_{11}\right)$ mode of the nanofiber has no axial component of the electric field at $r=0$.

For comparison, we calculate the coupling efficiency of the radiation from a source embedded at the center of a continuous

a

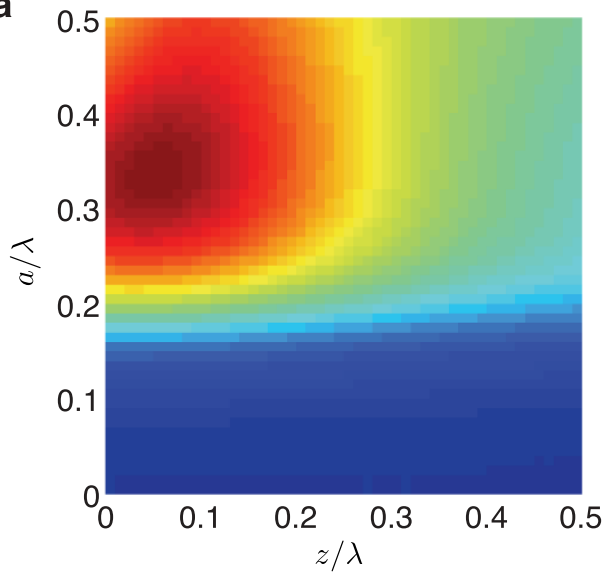

C

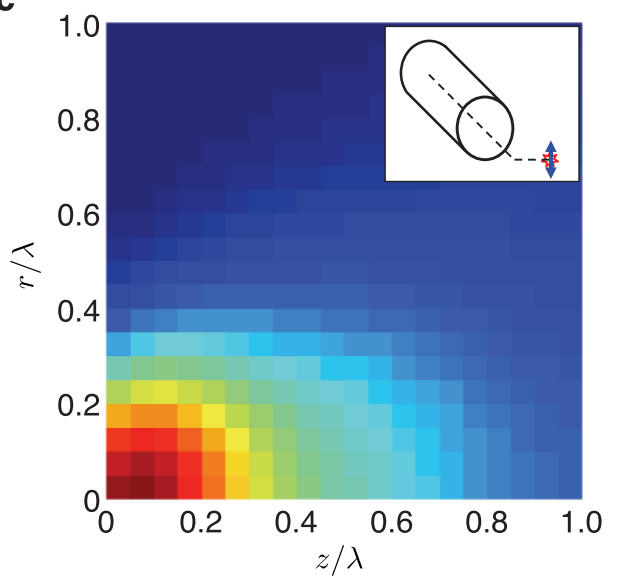

nanofiber of radius $a$. Figure $2 \mathrm{~b}$ shows the dependence of the coupling efficiency on the fiber radius $a$. (A continuous fiber has two output ports, and the radiation from the source is coupled to each of the two counter-propagating modes with equal coupling efficiency. We calculate the coupling efficiency for one of the two modes as shown in the inset of Fig. 2b.) The maximum coupling efficiency is $27 \%$, which is smaller than that when the source is placed on the nanofiber tip surface. This fact can be understood by considering the effect of the mirror image of the source created by the tip surface. The electric field of the radiation from the mirror image, or the reflected field, and that from the source destructively interfere on the vacuum side. This interference distributes more of the power of the source radiation to the fiber side than to the vacuum side. Indeed, the calculation using the amplitude transmission coefficient $t=2 /(1+n)$ $\approx 0.81$ and the amplitude reflection coefficient $r=(1-n) /(1+n) \approx$ -0.19 for the vacuum-silica boundary gives the power distribution ratio of $n|t|^{2}:|1+r|^{2} \approx 3: 2$ between the fiber side and the vacuum side, which agrees well with the efficiencies obtained above.

The coupling efficiencies obtained above correspond to the ratio $\Gamma_{\text {guided }}^{(1)} / \Gamma_{\text {total }}$ of the decay rate into the fundamental guided mode, $\Gamma_{\text {guided }}^{(1)}$, to the total decay rate, $\Gamma_{\text {total }}$, and they do not directly give the amount of the enhancement (or inhibition) of the spontaneous emission (the Purcell effect). We also calculate the ratio of $\Gamma_{\text {total }}$ to the decay rate of a dipole in free space, $\Gamma_{\text {free }}$, and obtain $\Gamma_{\text {total }} / \Gamma_{\text {free }}=1.28$ for $a=0.32 \lambda$. That is, the spontaneous emission of the dipole is enhanced compared to that in free space.

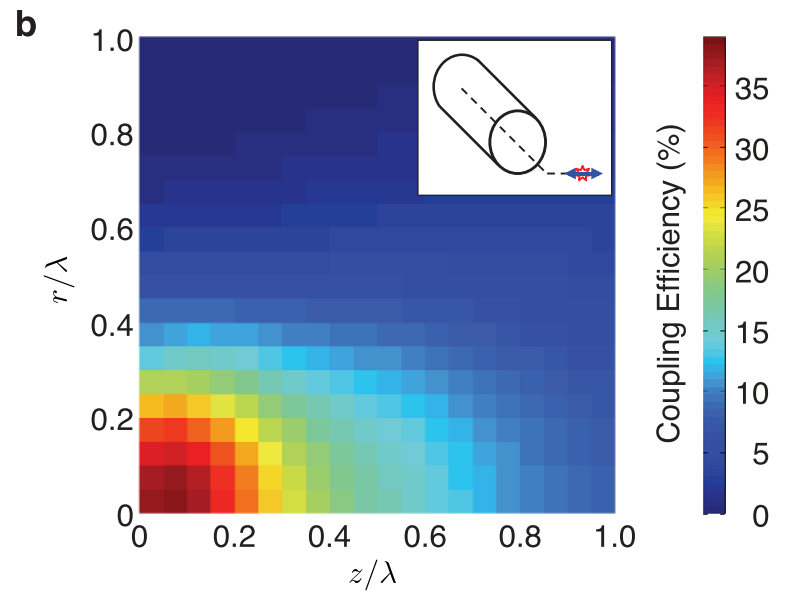

d
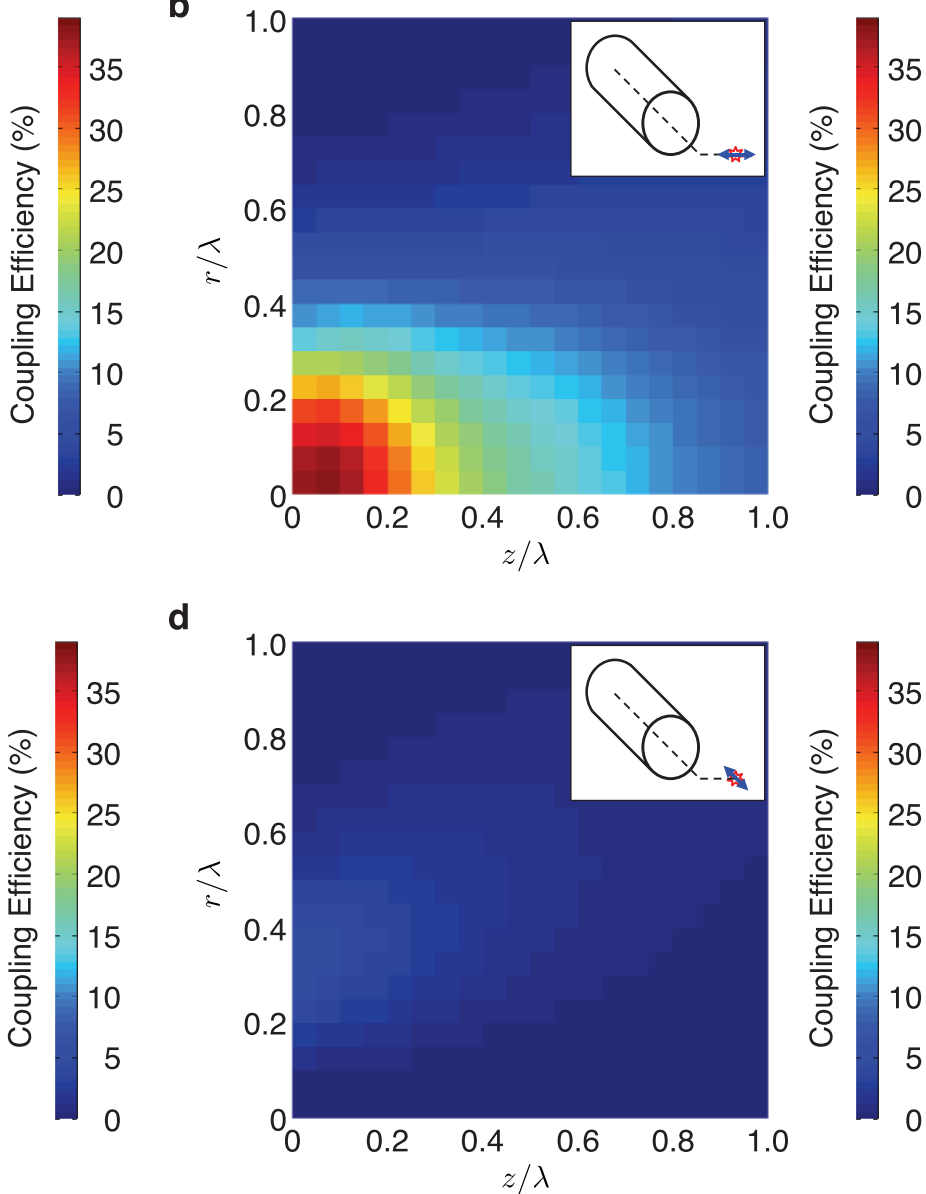

Figure $3 \mid$ Dependence of the coupling efficiency on the axial and radial positions of the source. (a), Dependence of the coupling efficiency on the nanofiber radius $a$ and the axial position $z$ of the point dipole source with radial polarization located on the fiber axis $(r=0)$. (b-d), Dependence of the coupling efficiency on $r$ and $z$ with a fixed fiber radius $a=0.32 \lambda$ for radial, azimuthal, and axial polarizations of the source, respectively. The insets show schematics of the spatial directions of polarizations. 
a

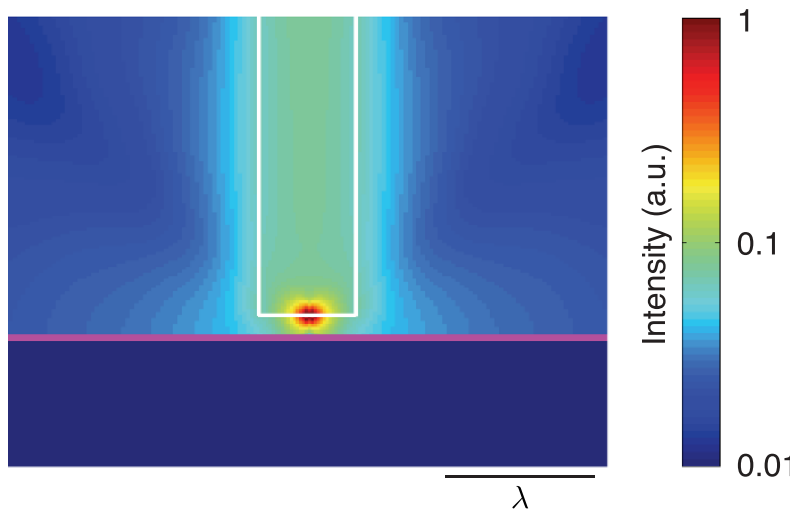

b

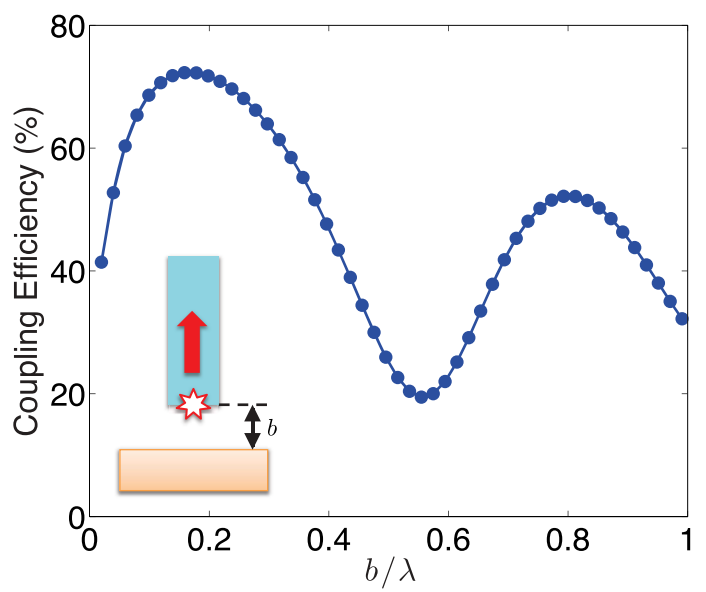

C

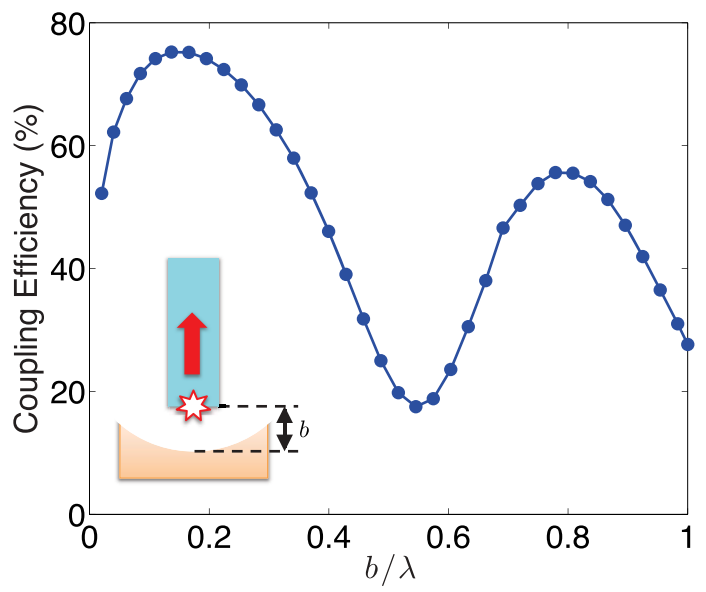

Figure 4 Increasing the coupling efficiency with the aid of a metallic mirror. (a), Calculated intensity of radiation for the case of a source position of $r=z=0$, a fiber radius of $a=0.32 \lambda$, and a tip-mirror distance of $b=0.15 \lambda$. The white and magenta lines represent the surfaces of the nanofiber and the metallic mirror, respectively. (b), Dependence of the coupling efficiency on the tip-mirror distance $b$, for the case of a flat mirror. The inset shows a schematic of the geometry. (c), Same as (b), for the case of a spherical mirror with a radius of curvature of $5 \lambda$.

The coupling efficiency of the radiation from a quantum emitter to the guided mode of a continuous nanofiber has been studied by several groups ${ }^{30,31,47}$. In order to compare the results obtained in the above calculation with these theories, we calculate the coupling efficiency based on the model in Ref. [31]. Specifically, the decay rate into the $i$-th guided mode and that into the radiation modes are respectively given by

$$
\begin{gathered}
\Gamma_{\text {guided }}^{(i)}=\frac{2 \omega_{0} \beta^{\prime}}{\varepsilon_{0} \hbar}\left|d \cdot e_{\text {guided }}^{(i)}\right|^{2}, \\
\Gamma_{\text {radiation }}=\frac{2 \omega_{0}}{\varepsilon_{0} \hbar} \sum_{m} \int_{0}^{k_{0}} d \beta\left|d \cdot e_{\text {rediation }}^{(\beta, m)}\right|^{2},
\end{gathered}
$$

where $\omega_{0}, k_{0}, \beta, \beta^{\prime}, \boldsymbol{d}, \boldsymbol{e}_{\text {guided }}^{(i)}, \boldsymbol{e}_{\text {radiation }}^{(\beta, m)}$ respectively denote the resonance frequency of the two-level atom, the vacuum wavenumber, the propagation constant, the derivative of $\beta$ with respect to the frequency $\omega$, the atomic dipole moment, the normalized electric field of the $i$-th guided mode, and the normalized electric field of the radiation mode with the propagation constant $\beta$ and the mode order $m$. The red solid line in Fig. $2 \mathrm{~b}$ shows the coupling efficiency, which is given by

$$
\frac{\Gamma_{\text {guided }}^{(1)}}{\Gamma_{\text {total }}}=\frac{\Gamma_{\text {guided }}^{(1)}}{\Gamma_{\text {radiaiton }}+\sum_{i} \Gamma_{\text {guided }}^{(i)}} .
$$

Its excellent agreement with our results clearly shows the validity of our FDTD calculations.

We next calculate the dependence of the coupling efficiency on the axial and radial position of the source. Figure 3 a shows the dependence of the coupling efficiency on $a$ and $z$ with a fixed radial source position $r=0$. The maximum coupling efficiency of $38 \%$ is obtained for $a=0.32 \lambda$ and $z=0.04 \lambda$. Figure $3 \mathrm{~b}-\mathrm{d}$ show the dependence of the coupling efficiency on $r$ and $z$ with a fixed fiber radius $a=0.32 \lambda$ for radial, azimuthal, and axial polarizations of the source, respectively. These results show that if a quantum emitter with a radial or azimuthal polarization is attached to or brought close to the center of the nanofiber tip with a position accuracy of $0.2 \lambda$, a coupling efficiency of $30-38 \%$ can be obtained.

The coupling efficiency can be increased with the aid of a mirror. We consider a flat metallic mirror oriented so that it is parallel to the face of the fiber, with a distance $b$ from the fiber tip. Figure 4a shows the calculated intensity of the radiation for the case of a source position of $r=z=0$, a fiber radius of $a=0.32 \lambda$, and a tip-mirror distance of $b=0.15 \lambda$. It shows that the radiation from the source is guided to the nanofiber more efficiently than in the case without a mirror (Fig.1c). Figure $4 \mathrm{~b}$ shows the dependence of the coupling efficiency on the tip-mirror distance $b$ for the case of $r=z=0$ and $a=0.32 \lambda$. The maximum coupling efficiency of $73 \%$ is obtained at $b=0.15 \lambda$. This can be slightly improved by using a spherical mirror; we obtain an efficiency of $76 \%$ for a mirror radius of curvature of $5 \lambda$, as shown in Fig. $4 c$.

\section{Discussion}

This scheme of using a nanofiber tip and a metallic mirror for coupling photons to the guided mode of a single-mode fiber can be applied to various kinds of quantum emitters. A semiconductor quantum dot or a diamond nanocrystal containing an NV center can be directly placed on the nanofiber tip surface in a manner similar to that in Refs. 41-43, where continuous nanofibers were used.

An atom can be laser-trapped by a dipole potential created by reddetuned light using the nanofiber tip, and high coupling efficiency of atomic emission to the fundamental guided mode of the nanofiber can be achieved. The van der Waals potential strongly shifts atomic energy levels in the vicinity of the surface of a dielectric or a metal within a distance of a few tens of nanometers ${ }^{48}$. In order to stably trap an atom with a dipole potential created by far-detuned laser light under practical conditions, its potential minimum has to be located at a distance of more than a few tens of nanometers from the surface. When a flat mirror is placed in front of the fiber tip, the output mode of the fiber forms a standing wave. For a tip-mirror distance of $0.4 \lambda \lesssim b \lesssim \lambda$, an anti-node of the standing wave is located around 


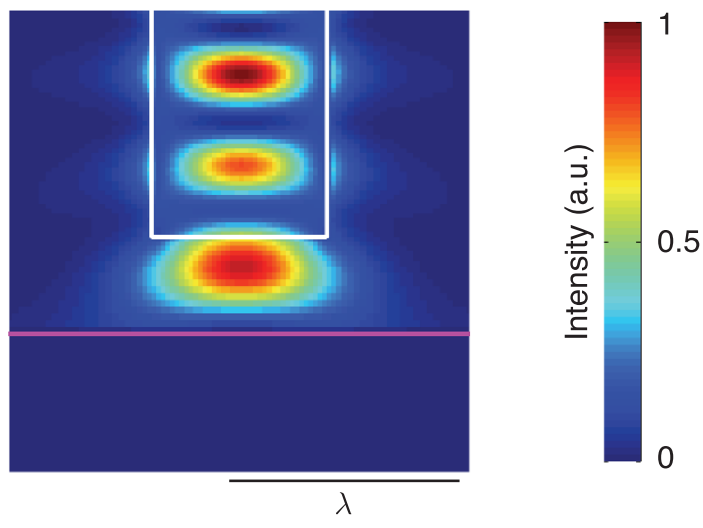

Figure $5 \mid$ Calculated intensity of the output mode of the nanofiber tip for the case of a wavelength of $\lambda=935 \mathrm{~nm}$, a fiber radius of $a=355 \mathrm{~nm}$, and a tip-mirror distance of $b=374 \mathrm{~nm}$. The white and magenta lines indicate the surfaces of the nanofiber and the metallic mirror, respectively.

the center between the tip and the mirror as shown in Fig. 5, which is suitable for creating a microscopic dipole trap ${ }^{49}$. In the case of a cesium atom, for example, laser light at the wavelength of $935 \mathrm{~nm}$ (the so-called magic wavelength ${ }^{50}$ ) with a power of $50 \mathrm{~mW}$ launched into the fundamental mode of a 355 -nm-radius nanofiber creates the trap minimum at a position $85 \mathrm{~nm}$ away from the tip when the tipmirror distance is $374 \mathrm{~nm}$, as shown in Fig. 6. For the radiation on the $6 \mathrm{~S}_{1 / 2} \mathrm{~F}=4 \rightarrow 6 \mathrm{P}_{3 / 2} \mathrm{~F}^{\prime}=5^{\prime}$ transition of a cesium atom at this trap minimum, the coupling efficiency to the fundamental guided mode of the nanofiber reaches $61 \%$. This is in stark contrast to the continuous nanofiber case, where the coupling efficiency for an atom trapped in a dipole potential created by a two-color evanescent field is less than $10 \%$ for each side of the guided mode ${ }^{36}$.

In a real experiment, there can be various imperfections. Although cleaving of a nanofiber to form a flat surface has been demonstrated $^{29}$, the shape of the nanofiber tip may be distorted from the ideally flat surface as considered above. In order to investigate the sensitivity of the coupling efficiency to the shape of the nanofiber tip,
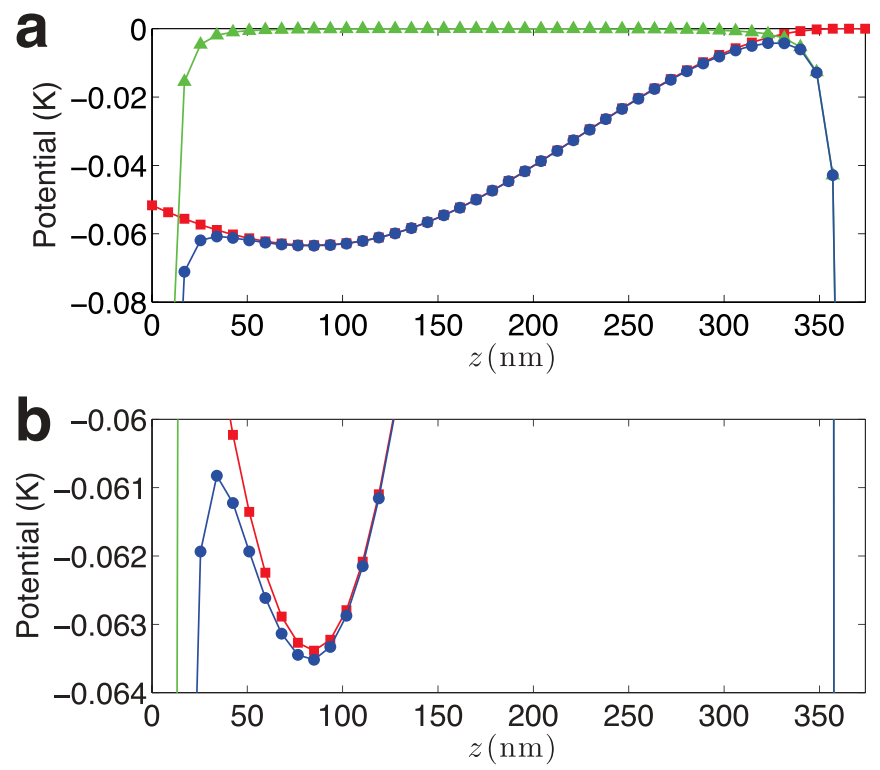

Figure $6 \mid$ (a) The red squares, the green triangles, and the blue circles represent the dipole potential $U_{\mathrm{d}}$ for laser light at the wavelength of $935 \mathrm{~nm}$ and an input power of $50 \mathrm{~mW}$, the van der Waals potential $U_{\mathrm{vdW}}$, and the total potential $U=U_{\mathrm{d}}+U_{\mathrm{vdW}}$, respectively, on the fiber axis $(r=0)$. (b) Same plot as (a) with a magnified vertical axis. we also calculate the coupling efficiencies for nanofibers with hemispherically shaped tips. Note that we have demonstrated fabricating nanofibers with hemispherical tips recently ${ }^{51}$. The obtained coupling efficiencies for $0<r / \lambda<1$ coincide with those for flat tips within $5 \%$. This indicates that the precise shape of the nanofiber tip does not strongly affect the coupling efficiency. Also, losses in the propagation in the device can be made negligible by fabricating low-loss tapered optical fibers. Indeed, fabrication of a tapered optical fiber with a subwavelength waist having a transmission in excess of $99.95 \%$ has been realized $^{34}$. It should be straightforward to apply the proposed device to, e.g., the recent experiments demonstrating the efficient coupling ( $22 \%$ in two ports) of photons from single quantum dots into the nanofiber-guided mode via the evanescent field ${ }^{43}$, and to experimentally achieve the high coupling efficiencies presented here.

The coupling efficiency can be even further increased by using a dielectric waveguide with a higher refractive index. For example, silicon has a refractive index of 3.5 and is transparent in the telecom wavelength. Here we consider a silicon waveguide with a square cross section. Figure 7 a shows the dependence of the coupling efficiency on
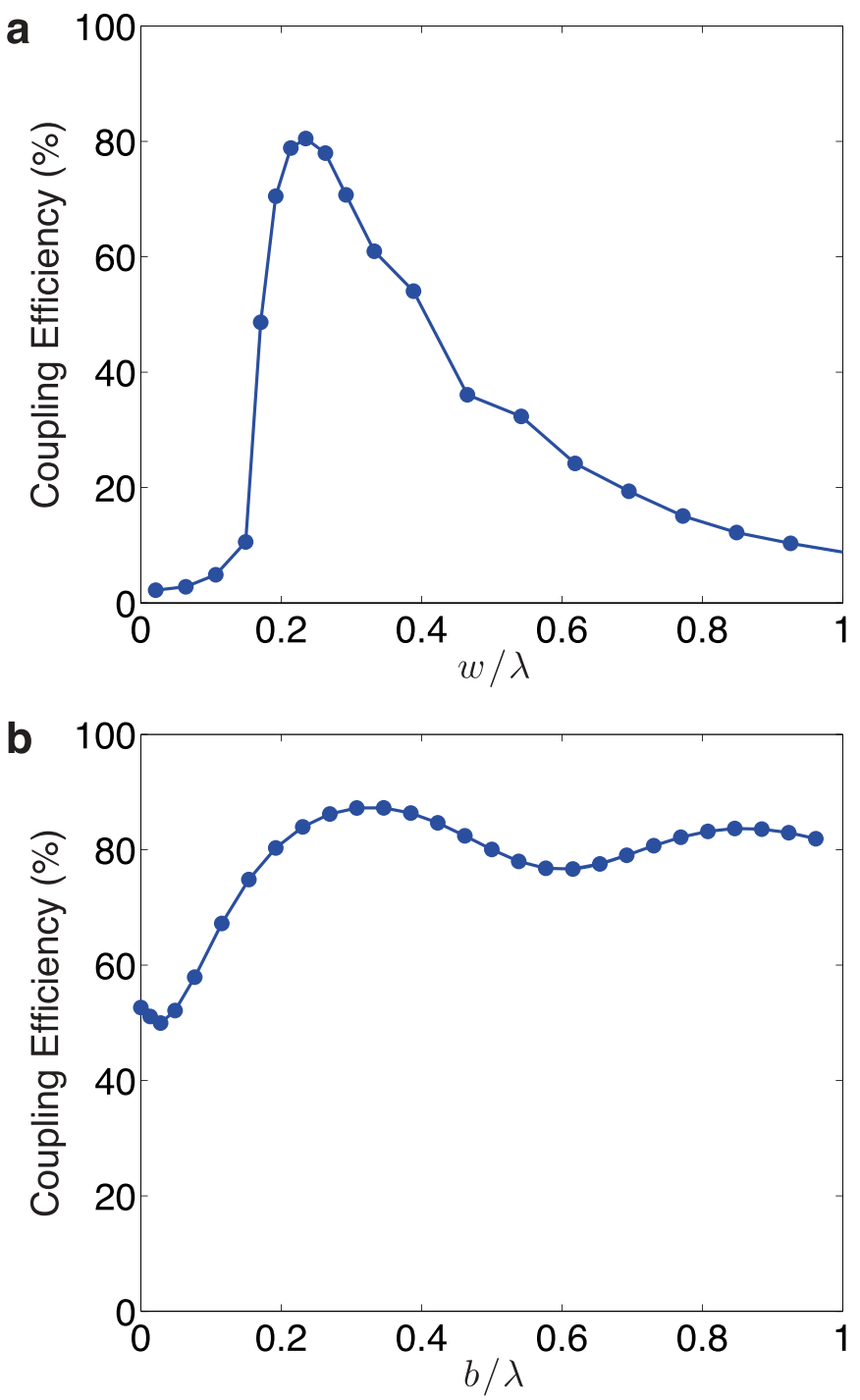

Figure $7 \mid$ Coupling efficiency of radiation from a point dipole source to the fundamental guided mode of the silicon waveguide with a square cross-section. a, Dependence of the coupling efficiency on the waveguide width $w$ when the source is attached to the center of the waveguide tip surface. $b$, Dependence of the coupling efficiency on the tip-mirror distance $b$ when the waveguide width of $w=0.235 \lambda$ and the source is attached to the center of the waveguide tip surface. 
the waveguide width $w$, with the location of the point dipole source fixed at the center of the waveguide tip surface. A maximum coupling efficiency of $80 \%$ is obtained at $w=0.235 \lambda$. When combined with a flat metallic mirror, the efficiency can be increased up to $87 \%$, as shown in Fig. 7 b. Such a silicon waveguide can be adiabatically coupled to a tapered optical fiber with low losses ${ }^{52}$.

In conclusion, we have studied a novel single-mode photon-coupling device utilizing a nanofiber tip. We have shown that a nanofiber with a flat tip can couple up to $38 \%$ and $76 \%$ of light from a point dipole source to a single-mode optical fiber without and with a mirror, respectively. When a silicon waveguide is used, a maximum coupling efficiency of $87 \%$ can be achieved. The proposed device directly couples photons from a quantum emitter to a standard single-mode optical fiber. In addition, the robust structure of this device is suitable for various experimental conditions including cryogenic temperature and ultra-high vacuum.

\section{Methods}

We employ a three-dimensional finite-difference time-domain method ${ }^{53}$ for the simulations. Figure $1 \mathrm{~b}$ is a schematic of the simulation geometry. We consider a silica nanofiber of radius $a$ and refractive index $n=1.46$, with one end cut flat along a plane normal to the fiber axis. A point dipole source is placed near the tip of the nanofiber, with an axial distance $z$ and a radial distance $r$ from the center of the nanofiber tip surface, respectively. We calculate the coupling efficiency of the radiation from the source to the fundamental guided mode of the nanofiber. The fundamental guided mode is numerically calculated using a mode solver.

1. Gisin, N., Ribordy, G., Tittel, W. \& Zbinden, H. Quantum cryptography. Rev. Mod. Phys. 74, 145-195 (2002).

2. Knill, E., Laflamme, R. \& Milburn, G. J. A scheme for efficient quantum computation with linear optics. Nature 409, 46-52 (2001).

3. Kok, P. et al. Linear optical quantum computing with photonic qubits. Rev. Mod. Phys. 79, 135-174 (2007).

4. McKeever, J. et al. Deterministic Generation of Single Photons from One Atom Trapped in a Cavity. Science 303, 1992-1994 (2004).

5. Darquie, B. et al. Controlled single-photon emission from a single trapped twolevel atom. Science 309, 454-456 (2005).

6. Keller, M., Lange, B., Hayasaka, K., Lange, W. \& Walther, H. Continuous generation of single photons with controlled waveform in an ion-trap cavity system. Nature 431, 1075-1078 (2004).

7. Lounis, B. \& Moerner, W. E. Single photons on demand from a single molecule at room temperature. Nature 407, 491-493 (2000)

8. Michler, P. et al. Quantum correlation among photons from a single quantum dot at room temperature. Nature 406, 968-970 (2000).

9. Santori, C., Fattal, D., Vučković, J., Solomon, G. S. \& Yamamoto, Y. Indistinguishable photons from a single-photon device. Nature 419, 594-597 (2002).

10. Kurtsiefer, C., Mayer, S., Zarda, P. \& Weinfurter, H. Stable solid-state source of single photons. Phys. Rev. Lett. 85, 290-293 (2000).

11. Miller, R. et al. Trapped atoms in cavity QED: coupling quantized light and matter. J. Phys. B 38, S551-S565 (2005).

12. Purcell, E. M. Spontaneous emission probabilities at radio frequencies. Phys. Rev. 69, 681 (1946).

13. Vahala, K. J. Optical microcavities. Nature 424, 839-846 (2003).

14. Aoki, T. et al. Efficient Routing of Single Photons by One Atom and a Microtoroidal Cavity. Phys. Rev. Lett. 102, 083601 (2009).

15. O'Shea, D., Junge, C., Volz, J. \& Rauschenbeutel, A. Fiber-Optical Switch Contrlled by a Single Atom. Phys. Rev. Lett. 111, 193601 (2013).

16. Srinivasan, K. \& Painter, O. Linear and nonlinear optical spectroscopy of a strongly coupled microdisk-quantum dot system. Nature 450, 862-865 (2007).

17. Hunger, D. et al. A fiber Fabry-Perot cavity with high finesse. New J. Phys. 12, 065038 (2010)

18. Steiner, M., Meyer, H. M., Deutsch, C., Reichel, J. \& Köhl, M. Single Ion Coupled to an Optical Fiber Cavity. Phys. Rev. Lett. 110, 043003 (2013).

19. Takahashi, H. et al. An integrated fiber trap for single-ion photonics. New J. Phys. 15, 053011 (2013)

20. Miguel-Sánchez, J. et al. Cavity quantum electrodynamics with charge-controlled quantum dots coupled to a fiber Fabry-Perot cavity. New J. Phys. 15, 045002 (2013).

21. Albrecht, R., Bommer, A., Deutsch, C., Reichel, J. \& Becher, C. Coupling of a Single Nitrogen-Vacancy Center in Diamond to a Fiber-Based Microcavity. Phys. Rev. Lett. 110, 243602 (2013).

22. Kaupp, H. et al. Scaling laws of the cavity enhancement for nitrogen-vacancy centers in diamond. Phys. Rev. A 88, 053812 (2013).

23. Lecamp, G., Lalanne, P. \& Hugonin, J. P. Very Large Spontaneous-Emission $\beta$ Factors in Photonic-Crystal Waveguides. Phys. Rev. Lett. 99, 023902 (2007).
24. Lund-Hansen, T. et al. Experimental Realization of Highly Efficient Broadband Coupling of Single Quantum Dots to a Photonic Crystal Waveguide. Phys. Rev. Lett. 101, 113903 (2008).

25. Thyrrestrup, H., Sapienza, L. \& Lodahl, P. Extraction of the $\beta$-factor for single quantum dots coupled to a photonic crystal waveguide. Appl. Phys. Lett. 96, 231106 (2010).

26. Chang, D. E., Sørensen, A. S., Hemmer, P. R. \& Lukin, M. D. Strong coupling of single emitters to surface plasmons. Phys. Rev. A 76, 035420 (2007).

27. Chang, D. E., Sørensen, A. S., Demler, E. A. \& Lukin, M. D. A single-photon transistor using nanoscale surface plasmons. Nature Phys. 3, 807-812 (2007)

28. Akimov, A. V. et al. Generation of single optical plasmons in metallic nanowires coupled to quantum dots. Nature 450, 402-406 (2007)

29. Tong, L., Zi, F., Guo, X. \& Lou, J. Optical microfibers and nanofibers: A tutorial. Opt. Commun. 285, 4641-4647 (2012).

30. Klimov, V. \& Ducloy, M. Spontaneous emission rate of an excited atom placed near a nanofiber. Phys. Rev. A 69, 013812 (2004).

31. Le Kien, F., Dutta Gupta, S., Balykin, V. I. \& Hakuta, K. Spontaneous emission of a cesium atom near a nanofiber: Efficient coupling of light to guided modes. Phys. Rev. A 72, 032509 (2005).

32. Love, J. D. \& Henry, W. M. Quantifying Loss Minimisation in single-mode fibre tapers. Electron. Lett. 22, 912-914 (1986).

33. Aoki, T. Fabrication of Ultralow-Loss Tapered Optical Fibers and Microtoroidal Resonators. Jpn. J. Appl. Phys. 49, 118001 (2010).

34. Ravets, S. et al. Intermodal energy transfer in a tapered optical fiber: optimizing transmission. J. Opt. Soc. Am. A 30, 2361-2371 (2013).

35. Sagué, G., Vetsch, E., Alt, W., Meschede, D. \& Rauschenbeutel, A. Cold-Atom Physics Using Ultrathin Optical Fibers: Light-Induced Dipole Forces and Surface Interactions. Phys. Rev. Lett. 99, 163602 (2007).

36. Nayak, K. P. et al. Optical nanofiber as an efficient tool for manipulating and probing atomic fluorescence. Opt. Express 15, 5431-5438 (2007).

37. Vetsch, E. et al. Optical Interface Created by Laser-Cooled Atoms Trapped in the Evanescent Field Surrounding an Optical Nanofiber. Phys. Rev. Lett. 104, 203603 (2010).

38. Das, M. et al. Measurement of fluorescence emission spectrum of few strongly driven atoms using an optical nanofiber. Opt. Express 18, 17154-17164 (2010).

39. Dawkins, S. T., Mitsch, R., Reitz, D., Vetsch, E. \& Rauschenbeutel, A. Dispersive Optical Interface Based on Nanofiber-Trapped Atoms. Phys. Rev. Lett. 107, 243601 (2011).

40. Goban, A. et al. Demonstration of a State-Insensitive, Compensated Nanofiber Trap. Phys. Rev. Lett. 109, 033603 (2012).

41. Fujiwara, M., Toubaru, K., Noda, T., Zhao, H.-Q. \& Takeuchi, S. Highly efficient coupling of photons from nanoemitters into single-mode optical fibers. Nano Lettt. 11, 4362-4365 (2011).

42. Schröder, T. et al. A nanodiamond-tapered fiber system with high single-mode coupling efficiency. Opt. Express 20, 10490-10497 (2012).

43. Yalla, R., Le Kien, F., Morinaga, M. \& Hakuta, K. Efficient Channeling of Fluorescence Photons from Single Quantum Dots into Guided Modes of Optical Nanofiber. Phys. Rev. Lett. 109, 063602 (2012).

44. Davanço, M. \& Srinivasan, K. Efficient spectroscopy of single embedded emitters using optical fiber taper waveguides. Opt. Express 17, 10542 (2009).

45. Davanço, M. \& Srinivasan, K. Fiber-coupled semiconductor waveguides as an efficient optical interface to a single quantum dipole. Opt. Lett. 34, 2542 (2009).

46. Srinivasan, K., Painter, O., Stintz, A. \& Krishna, S. Single quantum dot spectroscopy using a fiber taper waveguide near-field optic. Appl. Phys. Lett. 91, 091102 (2007).

47. Søndergaard, T. \& Tromborg, B. General theory for spontaneous emission in active dielectric microstructures: Example of a fiber amplifier. Phys. Rev. A 64, 033812 (2001).

48. Alton, D. J. et al. Strong interactions of single atoms and photons near a dielectric boundary. Nature Phys. 7, 159-165 (2010).

49. Schlosser, N., Reymond, G., Protsenko, I. \& Grangier, P. Sub-poissonian loading of single atoms in a microscopic dipole trap. Nature 411, 1024-1027 (2001).

50. McKeever, J. et al. State-Insensitive Cooling and Trapping of Single Atoms in an Optical Cavity. Phys. Rev. Lett. 90, 133602 (2003).

51. Kato, S., Chonan, S. \& Aoki, T. High-numerical-aperture microlensed tip on an air-clad optical fiber. Opt. Lett. 39, 773 (2014).

52. Gröblacher, S., Hill, J. T., Safavi-Naeini, A. H., Chan, J. \& Painter, O. Highly efficient coupling from an optical fiber to a nanoscale silicon optomechanical cavity. Appl. Phys. Lett. 103, 181104 (2013).

53. Taflove, A. \& Hagness, S. C. Computational Electrodynamics: The FiniteDiffernce Time-Domain Method, (3rd ed., Artech House, Inc., 2005).

\section{Acknowledgments}

This work is partially supported by SCOPE (111507004) and MATSUO FOUNDATION.

\section{Author contributions}

T.A. conceived and led the study. S.C. and S.K. carried out the calculations and analyzed data. T.A. prepared the manuscript. All authors reviewed the manuscript. 


\section{Additional information}

Competing financial interests: The authors declare no competing financial interests.

How to cite this article: Chonan, S., Kato, S. \& Aoki, T. Efficient Single-Mode

Photon-Coupling Device Utilizing a Nanofiber Tip. Sci. Rep. 4, 4785; DOI:10.1038/ srep04785 (2014) (i) This work is licensed under a Creative Commons Attribution-NonCommercialNoDerivs 3.0 Unported License. The images in this article are included in the article's Creative Commons license, unless indicated otherwise in the image credit; if the image is not included under the Creative Commons license, users will need to obtain permission from the license holder in order to reproduce the image. To view a copy of this license, visit http://creativecommons.org/licenses/by-nc-nd/3.0/ 